\title{
A comparison of in vitro fertilization-embryo transfer outcome by two types of soft embryo transfer catheters
}

\author{
Soghra Rabiei ${ }^{1}$, Mahnaz Yavangii ${ }^{2}{ }^{*}$, Marzieh Farimani ${ }^{3}$, Iraj Amiri ${ }^{4}$, Mohamad Fallah ${ }^{5}$, Hasan Bahrami ${ }^{6}$, \\ Shahedeh Khansari ${ }^{7}$
}

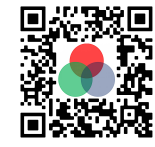

Use your smartphone to scan this QR code and download this article

${ }^{1}$ School of Medicine, Hamadan University of Medical Sciences, Hamadan, Iran

${ }^{2}$ Department of Obstetrics and Gynecology, School of Medicine, Endometrium and Endometriosis Research Center, Fatemieh Hospital, Hamadan University of Medical Sciences, Hamadan, Iran

${ }^{3}$ Endometrium and Endometriosis Research Center, Hamadan University of Medical Sciences, Hamadan, Iran

${ }^{4}$ School of Medicine, Endometrium and Endometriosis Research Center,

Hamadan University of Medical

Sciences, Hamadan, Iran

${ }^{5}$ School of Medicine, Endometrium and Endometriosis Research Center, Hamadan University of Medical Sciences, Hamadan, Iran

\section{Correspondence}

Mahnaz Yavangi, Department of Obstetrics and Gynecology, School of Medicine, Endometrium and

Endometriosis Research Center,

Fatemieh Hospital, Hamadan University

of Medical Sciences, Hamadan, Iran

Email:dr_sh_kh@yahoo.com History

- Received: Jan 07, 2020

- Accepted: Mar 05, 2020

- Published: Mar 30, 2020

DOI : 10.15419/bmrat.v7i3.594

\section{Check for updates}

\section{Copyright}

(C) Biomedpress. This is an openaccess article distributed under the terms of the Creative Commons Attribution 4.0 International license.

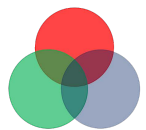

\begin{abstract}
Introduction: One of the remaining challenges in assisted reproductive procedures, especially in vitro fertilization (IVF), is proper embryo transfer. The aim of this clinical trial was to compare IVFembryo transfer outcome by two types of soft embryo transfer catheters in Hamadan Endometrics and Endometriosis Research Center (Iran). Methods: In this clinical trial study, 100 patients who were candidates for IVF were evaluated. Patients were randomly assigned into two groups $(A=50$ and $B=50)$. The IVF was identical for both groups until the embryo transfer stage. For group A, soft catheter CH3 PM TRANS SET MINI was used and in group B, KITAZATO soft catheter was used for embryo transfer. All transfers were performed by one person. Patients were recruited using checklists, demographic information, infertility history, beta-human chorionic gonadotropin (ß-hCG) serum levels at day 14 post-transfer, and pregnancy bag 28 days after transfer. The results were analyzed by SPSS software version 16 and using descriptive statistics, chi-square and t-test. The significance level was $<0.05$. Results: The mean age of group A and group B was 30.12 and 29.24 years, respectively ( $p=0.341)$. The mean duration of infertility in both groups was not statistically significant, and in groups A and B were 4.89 and 4 years, respectively. Ninety \% of group A experienced their first IVF experience, while in group B it was slightly lower than $86 \%$, which was not statistically significant. The mean number of eggs obtained in group A was 9.84 and in the group B was $9.88(p=0.962)$. The mean number of embryos formed in group $A$ was 6.24 and in group B was $5.72(p=0.405)$. There was no statistically significant difference between the two groups in using of Tenaculum, the quality of transmission, and the contamination of the catheter head into the blood or mucus. Conclusion: According to the findings of the present study, the use of KITAZATO catheter compared to PM TRANS SET MINI CH3 catheter for fetal transfer in IVF patients showed no significant difference in pregnancy success rate. However, patients who received the KITAZATO catheter had a slightly higher chance of pregnancy that could be clinically valuable.
\end{abstract}

Key words: In vitro fertilization, Embryo Transfer, Catheters, Pregnancy rate

\section{INTRODUCTION}

Almost 10 to $15 \%$ of couples have infertility, which is defined as the absence of pregnancy after one year of unprotected sex ${ }^{1}$. The main causes of infertility include male factor, decreased ovarian reserve, ovulation abnormalities, tubal injury, obstruction or adhesion around the tube, uterine factor, systemic diseases, cervical agents, immunologic factors, and unexplained factors ${ }^{2}$. Over the past few decades, successful therapies have been developed for all causes of infertility, which has given hope to couples with this medical problem ${ }^{1}$. Assisted Reproductive Technologies (ART) includes all the methods used to directly manipulate the eggs outside the body. The first form of ART that is still the most common is in vitro fertilization (IVF) ${ }^{3}$. ART includes IVF, Intra-cytoplasmic Sperm Injection (ISCI), Gamete intra fallopian transfer (GIFT), Zygote intra fallopian transfer (ZIFT), and use of donor oocytes (which has been reduced due to improved IVF embryo transfer success, GIFT and ZIFT).

ART focuses on IVF and ISCI. Both of these technologies include ${ }^{2}$ : Prevention of LH surge, follicle growth, pre-treatment, ancillary drugs, oocyte maturation / ovulation, oocyte harvest, luteal phase support, fertilization, laboratory culture of ambrosia, transfer of fresh embryo, freeze of extra embryos, and first trimester pregnancy monitoring. Although most patients who reach the embryo transfer stage have good quality embryos, it is actually the stage of transmission that determines the success or failure of $\mathrm{IVF}^{4}$. The purpose of embryo transfer through the cervix is to deliver the embryo to the optimum location of the uterus for implantation without trauma ${ }^{2}$. Many factors may influence the success of embryo transfer, including the technique and experience of the operator, the type of catheter, the size and nature of the catheter, 
${ }^{6}$ Department of Parastilogy and Mycology, School of Medicine, Hamadan University of Medical Sciences, Hamadan, Iran

${ }^{7}$ School of Medicine, Hamadan University of Medical Sciences, Hamadan, Iran bleeding, uterine muscles, catheter transfer, and retention process ${ }^{4}$. The main factors affecting embryo implantation include: the strength of uterus, the quality of uterus, and the efficiency of transfer process ${ }^{4}$. Embryo transfer is the last difficult step in the IVF process and, thus, the type of catheter chosen is crucial $^{5}$.

Transmission catheters have a wide variety of designs. They may be hard or completely soft. The hard catheters are easier to insert but may cause trauma and uterine contractions more than soft catheters. The soft catheters are barely inserted into the cervix, require special instruments (Tenaculum) to be inserted, and are associated with greater clinical pregnancy rates ${ }^{3}$. The operator determines which transfer was difficult versus easy. The catheter is inserted easily into the uterine cavity without any problems. However, for difficult transmission, it takes a long time for the catheter to be inserted into the uterine cavity; it also requires the use of tenaculum and sometimes catheter replacement ${ }^{5}$. Contamination of blood and mucus transfer on catheters during embryo transfer is both difficult yet easy to transfer, but is more associated with difficult transmission and only has a negative effect on pregnancy rate during hard transfer ${ }^{6}$. There have been limited studies comparing the effects of catheters on IVF results and in some cases conflicting results are available. The purpose of this clinical trial was to compare IVF-embryo transfer outcome by two types of soft embryo transfer catheters in Hamadan Endometrics and Endometriosis Research Center in 2015 in Hamadan, Iran.

\section{METHODS}

This study was a randomized controlled clinical trial. Patients were selected from those who had been referred to The Endometrial and Endometriosis Research center of Hamadan (Iran) due to infertility problems and those who were candidates for IVF embryo transfer. Patients with the same cause of infertility were selected and patients whose causes of infertility were not significantly affected by the study were also selected. Based on the results of the study by Meriano J and colleagues ${ }^{4}$, the sample size in each group was 45 patients. However, in our study, 50 patients were studied in each group for greater accuracy and probability of withdrawal (Figure 1). The inclusion criteria were: patients who were less than 40 years old, more than 3 embryos for transfer, patients with a healthy uterine cavity with hysterosalpingography and hysteroscopy, patients with fixed embryo culture medium, patients with stable fetal quality, patients with stable embryo transfer environment, patients with a fixed embryologist, patients with a stable gynecologist from the beginning of the IVF to the end, and patients with a fixed number of transient embryos (considered in this study to be three embryos). The exclusion criteria were: patients who are candidates for the follicle-stimulating hormone (FSH) high baseline transfer process, patients with a history of difficult transmission in the previous procedure, patients who have had an IVF cycle more than 6 times, patients with causes of idiopathic infertility, patients who did not sign the moral consent form, patients with donated oocytes, and patients whose embryos are frozen.

\section{Classification of groups}

Ninety patients were selected from eligible patients who were selected according to inclusion and exclusion criteria, and accepted according to the inclusion consent. They were randomly divided into two groups $(A=45$ and $B=45)$. The IVF of both groups was identical to the embryo transfer stage. In group A, PM TRANS SET MINI CH3 soft catheter was used, and in Group B, KITAZATO soft catheter was used for group B's embryo transfer. All transfers were performed by one person to prevent confounding factors. Patients were referred (on the 14th day after transplantation) for serum beta-human chorionic gonadotropin ( $B$ hCG), and then on the 28th day after transplantation for sonographic sac examination.

\section{Retrieval}

Retrieval was done by vaginal ultrasound about 2 to 3 hours after $ß$-hCG injection. This was performed in the operating room by transvaginal ultrasound via follicle aspiration. In the laboratory, after blood and cell removal, granulosa oocysts scrapes were extracted.

\section{Oocyte cultivation}

For embryo culture, a variety of environments were used. Most of the media used for IVF contain a protein source, such as maternal serum, fetal cord serum, albumin or serum globulin.

\section{Assisted Reproduction Technology}

IVF consists of a fully coordinated sequence of actions that begins with vigorous and controlled ovarian stimulation with ovarian gonadotropins followed by transvaginal ultrasound monitoring of oocyte recycling. After fertilization in the laboratory, the embryos are transplanted into the uterus transversely. 


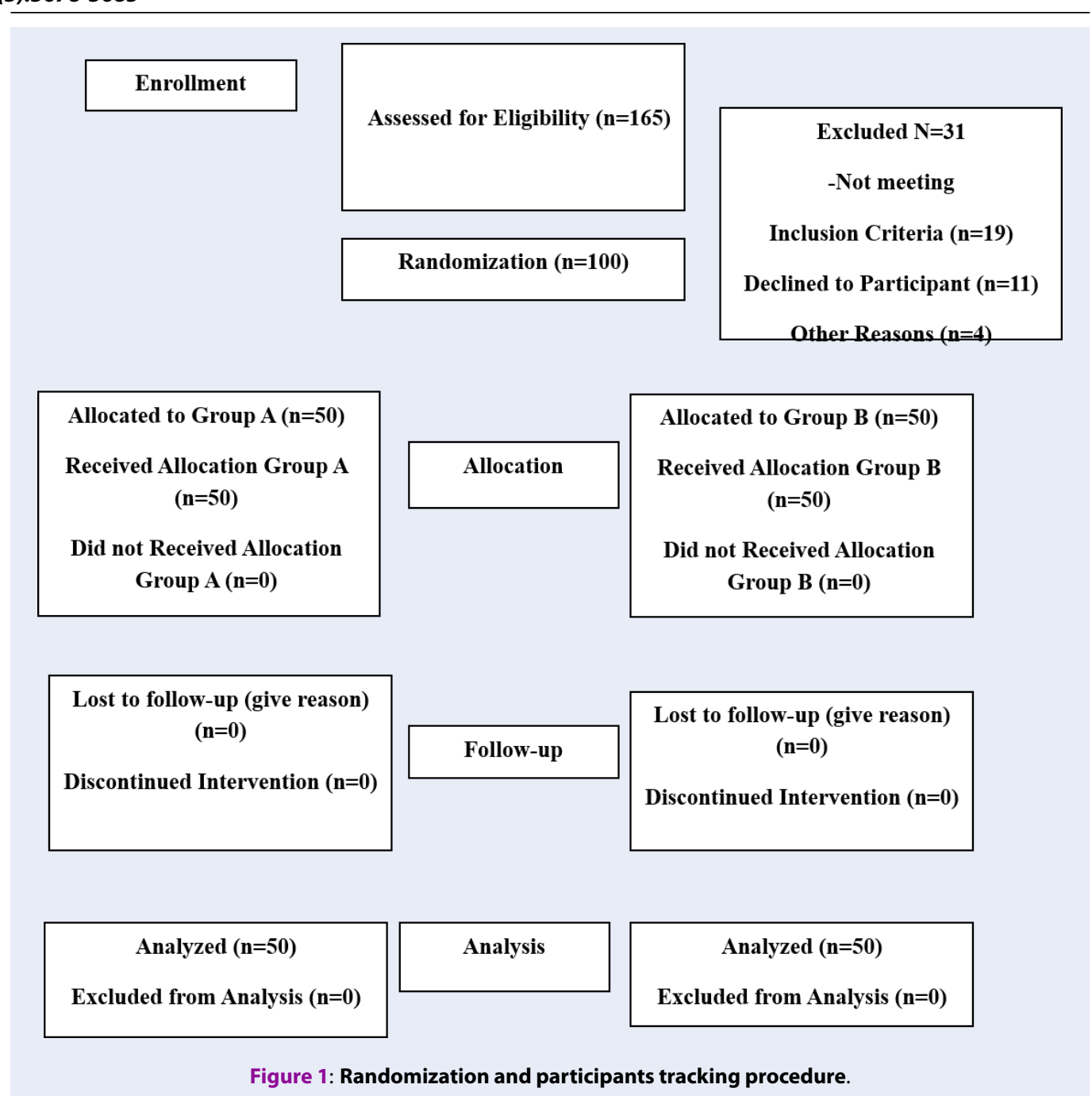

Early IVF experiences were experienced in women with tubal disease. However, in the early 1990s, this treatment expanded to a male-induced, unexplainable immunological cause of infertility or decreased ovarian function .

Embryo transfer is the last difficult step in IVF; the choice for type of catheter is very crucial. Transfer catheters can vary greatly in design type; for example, they may be hard or soft. Hard catheters cause trauma and uterine contractions. Soft catheters are barely inserted into the cervix, require special tenaculum insertions, and are associated with greater clinical pregnancy rates.

\section{Embryo transfer}

Embryo transfer has been successfully performed at every step from the pre-nuclear stage to the blastocyst. However, embryos are most commonly transferred when they are evolutionarily between the 8-10 cell stages (approximately 72 to 80 hours after oocyte retrieval).

Subsequent therapies (such as prostaglandin inhibitors, sedatives, and antibiotics) around the time of transfer are not of proven value. One of the most important issues in embryo transfer is embryo grading. Grade 1 embryos have 8 identical cells and less than 15\% fragmentation. Grade 11 embryos have 6 to 8 cells identical to $25 \%$ fragmentation. Grade III cells contain less than 6 cells with a fragment of severe saddle.

\section{Blinding of the study}

The study was single-blinded. Ideally, the study would be such that the infertility specialist would not aware of the type of catheter (type of intervention). However, the appearance of the catheter made it such that the type could be determined so it was not possible for the operator using the catheter to be unaware; 
there was practically no way to hide it from the operator. The type of catheter can only be kept secret from the operator until the moment of embryo transfer, such that the operator's mental background of the catheters would not affect his/her performance during the transfer. Patients remained unaware of the type of catheter used during the transfer until the end of the procedure, avoiding problems such as skipping the study.

\section{Defining outcomes}

The outcomes of the study were defined as primary and secondary. The primary outcome was: clinical pregnancy rate (which was defined as and determined by pregnancy test positive with $\beta$-hCG titers above 20 units at day 14 after transfer, subsequent pregnancy with uterine sac determined by trans-vaginal ultrasound at week 4 of transmission, ectopic pregnancy, and positive $B-h C G)$.

The secondary outcome was: rate of difficult transfer (which was defined as a time-consuming transient process with the need for tenaculum or catheter replacement). After completing the catheter transfer procedure, the fetus was checked to confirm the presence of blood or mucus.

\section{Control group}

Since KITAZATO soft catheter is routinely used in the process of embryo transfer in infertility treatment centers, group B was considered as the control group and group $\mathrm{A}$ as the experimental group.

\section{Instruments}

The data collection tool consisted of two sections of baseline information and data from the current study. Questions such as type of infertility, duration of infertility, number of previous IVFs, parity type and abortion histories were used to assess baseline information. Also, to assess the effects of the present study, questions and data collected also included: number of oocyte retrieval, number of embryos formed, quality of embryos formed, number of embryos transferred, type of catheter used in the embryo transfer process, ß-hCG test positive (titers above 20 units) on day 14 after embryo transfer, observation of pregnancy with ultrasound on day 28 after embryo transfer, use of tenaculum during fetal transfer, catheter replacement during fetal transfer, and observation of blood or mucus in catheter during fetal and qualitative transfer. The study was conducted in coordination with Hamadan University of Medical Sciences in Iran. Study data were collected without mentioning individual and demographic characteristics and the results were generally reported. Written consent was obtained from the participants prior to the study. This study was approved by the Ethical Committee of Hamadan University of Medical Sciences (IR.UMSHA.REC.1394.369).

\section{Data analysis}

Data analysis was performed using SPSS version 22 . Descriptive and chi-square tests were used to compare the mean of the intervention effect between the two groups. The significance level of $<0.05$ was deemed to be statistically significant in data comparisons.

\section{RESULTS}

In this clinical trial study, 100 patients who were candidates for IVF surgery were studied. In the embryo transfer phase, PMTRANSSETMINI CH3 soft catheter was used in group A and in group B, KITAZATO soft catheter was used. We briefly named the groups as A and B in this study for ease of comparison. The mean $( \pm S D)$ ages of participants in group A and group B were $30.12( \pm 4.50)$ and 29.24 $( \pm 4.69)$, respectively. Also, the mean $( \pm S D)$ duration of infertility in group A versus group B was 4.89 $( \pm 4.17)$ and $4( \pm 3.67)$, respectively. The results of this study showed that between two groups, with respect to age $(p=0.341)$ and duration of infertility $(p=0.260)$, these variables were not statistically significant different (Table 1). Also, the results of chi-square test showed that there were no significant differences between groups A and B in terms of variables such as infertility $(\mathrm{p}=0.534)$, number of IVF cycles $(\mathrm{p}=538)$, parity $(\mathrm{p}=0.398)$, and abortion $(\mathrm{p}=0.799)$ (Table 2$)$. The mean $( \pm \mathrm{SD})$ number of oocytes in group A versus group B was $9.84( \pm 4.46)$ and $9.88( \pm 4.05)$, respectively. There was no statistically significant difference in the mean number of oocytes between the two study groups $(\mathrm{p}=0.962)$. Also, the mean $( \pm \mathrm{SD})$ number of embryos in group A and group B was $6.24( \pm 3.53)$ and $5.72( \pm 2.61)$, respectively. The number of embryos ready for transfer in both groups was not statistically significant (Table 3$)(\mathrm{p}=0.405)$. According to the findings of this study, the frequency of positive chemical $(A=12, B=20)$ and clinical pregnancy $(A=11$, $B=19)$ in group $B$ was higher than that of group $A$, respectively, though not statistically different when comparing the groups for those variables $(\mathrm{P}=0.086$ and $\mathrm{p}=0.081$, respectively) (Table 4 ). No catheter replacement was required in any of the patients. The use of tenaculum and embryo transfer quality was similar in both groups. Blood was detected in the catheter head only in 5 patients, 3 of which belonged to group A $(\mathrm{P}=0.646)$ (Table 4). 
Table 1: The comparison of mean (SD) age and duration of infertility between two groups $(A=50, B=50)$

\begin{tabular}{lccc}
\hline \multicolumn{1}{c}{ Variables } & Mean & SD & $p$-value \\
Age & & & 0.341 \\
Group A & 30.12 & 4.50 & \\
Group B & 29.24 & 4.69 & 0.260 \\
Duration of Infertility & & & \\
Group A & 4.89 & 4.17 & \\
Group B & 4 & 3.67 & \\
\hline
\end{tabular}

Table 2: Comparison of demographics variables between two groups

\begin{tabular}{lccc}
\hline Variables & $\begin{array}{c}\text { Group A } \\
\text { N (\%) }\end{array}$ & $\begin{array}{c}\text { Group B } \\
\text { N (\%) }\end{array}$ & 0.534 \\
Type of infertility & & & \\
Primitive & $33(66)$ & $30(60)$ & 0.799 \\
Secondary & $17(34)$ & $20(40)$ & \\
Abortion & & & \\
Yes & $10(20)$ & $9(18)$ & 0.538 \\
No & $40(80)$ & $41(82)$ & \\
IVF Cycles & & $43(86)$ & \\
First time & $45(90)$ & $7(14)$ & \\
More than once & $5(10)$ & & \\
Parity & & $31(62)$ & \\
Nolipara & $35(70)$ & $50(100)$ & \\
Multipara & $15(30)$ & $50(100)$ & \\
Total & &
\end{tabular}

Table 3: Comparison of the mean (SD) oocytes and embryos formed between the two groups

\begin{tabular}{lccccc} 
& \multicolumn{2}{c}{ Group A } & \multicolumn{2}{c}{ Group B } & $p$-value \\
Variable & Mean & SD & Mean & SD & \\
oocytes & 9.84 & 4.46 & 9.88 & 4.05 & 0.962 \\
embryos & 6.24 & 3.53 & 5.72 & 2.61 & 0.405 \\
\hline
\end{tabular}

\section{DISCUSSION}

The purpose of this clinical trial was to compare IVFembryo transfer outcome by two types of soft embryo transfer catheters in the Hamadan Endometrics and Endometriosis Research Center. Assisted reproductive technology methods have greatly improved in recent decades, leading to increased gestational success in these patients. Studies published in recent years show that embryo transfer techniques have a great impact on the success of this procedure and physicians pay more attention to it. Although many factors influence the choice of catheter type, individual experience and training have a great impact on the choice of catheter $^{7,8}$. One of the study objectives was to compare the chemical pregnancy and clinical pregnancy rates between group $\mathrm{A}$ and group $\mathrm{B}$.

The findings of our study showed that chemical pregnancy and clinical pregnancy rates were higher in group B than in group A. Twelve patients in group A and 20 in group B were observed after 14 days of 


\begin{tabular}{lccc}
\hline Table 4: Comparison of pregnancy outcome in two study groups & & \\
\hline Fertility & $\begin{array}{c}\text { Group A } \\
\text { N (\%) }\end{array}$ & $\begin{array}{c}\text { Group B } \\
\text { N (\%) }\end{array}$ & $\mathbf{p}$-value \\
Chemical & & & \\
Yes & $12(24)$ & $20(40)$ & \\
No & $38(76)$ & $30(60)$ & $\mathbf{0 . 0 8 1}$ \\
Clinical & & & \\
Yes & $11(22)$ & $19(38)$ & \\
No & $39(78)$ & $31(62)$ & \\
\hline
\end{tabular}

Table 5: Comparison of complications and quality of embryo transfer in two study groups

\begin{tabular}{lccc}
\hline Variable & $\begin{array}{c}\text { Group A } \\
\text { N (\%) }\end{array}$ & $\begin{array}{c}\text { Group B } \\
\text { N (\%) }\end{array}$ & $\mathbf{p}$-value \\
Tenaculum & & & $\mathbf{1 . 0 0}$ \\
Yes & $1(1)$ & $1(1)$ & \\
No & $49(98)$ & $49(98)$ & $\mathbf{0 . 6 4 6}$ \\
Blood & & & \\
Yes & $3(6)$ & $2(4)$ & \\
No & $39(78)$ & $31(62)$ & $\mathbf{1 . 0 0}$ \\
Quality & & & \\
Hard & $1(1)$ & $49(1)$ & \\
EASY & $49(98)$ & $48)$ & \\
\hline
\end{tabular}

embryonic transfer of $\beta$-hCG. The observed difference was not statistically significant but from a clinical point of view, it could be considered significant if the study had a larger statistical volume. The same situation was observed for the parameter of clinical pregnancy, which was observed in $22 \%$ of patients in group A and $38 \%$ of those in group B.

Past studies have also shown that there is no significant difference between soft catheters in pregnancy success rates. In a study by Myvanwy Mcllveen et al., the results showed that there was no statistically significant difference in the odds of clinical pregnancy success using the two soft catheters (Edwards Wallace's catheter and Cook's catheter $)^{9}$. In a meta-analysis conducted by Abou Setta, A and colleagues, the results showed that using a soft catheter, although more difficult than a soft catheter, increased the chance of pregnancy ${ }^{7}$. In a meta-analysis performed by Zhan, Y et al., the results showed that soft catheters did not differ in the rate of pregnancy success, but the person performing the procedure has the ability and experience to influence the success rate ${ }^{10}$. In another study by Tiffany L. Rhodes and colleagues, the results showed that Cook's and Edwards Wallace's catheters (both of which are soft), did not differ significantly in induction of clinical pregnancy rates ${ }^{11}$. One of the theories that demonstrates and justifies the greater results with a soft catheter than a hard catheter is that soft catheters do less damage to the endometrium and cause fewer contractions in the uterus following the use of the catheter. In the present study, all embryo transfers were performed by one person. Thus, the results of our study was not affected by multiple operators, although the performance of the individual at different times may be affected.

Some of the study objectives were to evaluate the effect of soft catheters in both groups on the use of tenaculum, the complexity of transfusion, and the observation of blood and mucus in the catheter head. As expected, with the use of soft catheters in both groups, there was no observed statistically significant difference in the use of tenaculum, the complexity of transfusion, or the observation of blood and mucus in the catheter head. There was not any samples from two groups to indicate that additional costs were imposed economically. One of the drawbacks 
of using soft catheters is their more complicated technique and failure, which sometimes requires the use of instruments such as the tenaculum for fetal transfer, and even in some cases, it is not possible to use soft catheters. In a study by Wood et al. which looked at 867 fetuses transplanted with a soft catheter, 1.3 cases were unenforceable and $3.2 \%$ required cervical dilatation or more than 5 minutes for transfer ${ }^{12}$. In our study, $2 \%$ of the operator cases required the use of tenaculum, which was similar in both groups, and which was less than the rate mentioned above. In a study conducted by Pia Saldeen et al., the results showed that the Edwards Wallace catheter was replaced due to difficulty in transposition, which in our study showed no difference in catheter dilation ${ }^{13}$. The presence of blood in the catheter or catheter allograft is one of the factors that reduces the chance of pregnancy in IVF patients ${ }^{14}$.

Based on the findings of the present study, the mean age, type of infertility, duration of infertility, IVF experience, abortion, and parity were not significantly different between groups $\mathrm{A}$ and $\mathrm{B}$, indicating that the results of the present study could not be influenced by demographic or baseline variables. Finally, it is important to note that most IVF and ICSI candidates reach a stage where embryo quality is favorable for embryo transfer but, unfortunately, the implantation and success of these methods is not optimal. The risk of embryo displacement reduces the risk of damage to the cervix and endometrium that may lead to pregnancy. Although soft catheters may cause damage to the endometrial catheter, they may increase the chances of pregnancy in patients and may be an appropriate choice for patients, as they are less likely to cause endometrial injury than other catheters. One of the limitations of the present study was that the present study only examined patients who were referred to our study center, so patients who did not visit this center may be different from our patients.

\section{CONCLUSIONS}

According to the findings of the present study using the KITAZATO catheter compared to the PM TRANS SET MINI CH3 catheter for fetal transfer in IVF patients, there was no significant difference in pregnancy success rate. However, patients who received the KITAZATO catheter had a slightly higher chance of pregnancy that could be clinically valuable. It is generally recommended that a similar study with a larger sample size be performed to compare the performance of the soft and hard catheters in the future.

\section{ABBREVIATIONS}

ART: Assisted Reproductive Technologies

IVF: In Vitro Fertilization

ICSI: Intra cytoplasmic Sperm Injection

GIFT: Gamete intra fallopian transfer

ZIFT: Zygote intra fallopian transfer

\section{AUTHORS' CONTRIBUTIONS}

All authors contributed equally in the study design, interpretation of the data, and writing of the final manuscript.

\section{COMPETING INTERESTS}

The author(s) declare that they have no competing interests.

\section{ACKNOWLEDGMENTS}

We thank the manager of Fatemieh hospital and also participants to participate in this study.

This study was supported by Hamadan University of Medical Sciences.

\section{REFERENCES}

1. Wright K, Johnson J. Infertility. in: Gibs RS, Karlyn BY, Haney AF, Nygaard IE. Danforth's bstetrics and Gynecology. Philadelphia: Lippincott Williams \& Wilkins10th ed. 2008;p. 705-715.

2. Aubuchon M, Burney O, Schust J, Yao M. Infertility and Assisted reproductive technology. In: Berek SJ. Berek and Novak's Gynecology. 15th ed. Philadelphia: Lippincott Williams \& Wilkins. 2012;p. 1133-1189.

3. Fitz M, Speroff L. Assisted reproductive technologies. in: Clinical Gynecologic Endocrinology and Infertility. 8th ed. Philadelphia: Lippincott Williams \& Wilkins. 2011;p. 13311382.

4. Meriano J, SC B, T R, Weissman A, Greenblatt M, Ward S, et al. The choice of embryo transfer catheter affects embryo implantation after IVF. Fertility and Sertility. 2000;74(4):678-682. Available from: https://doi.org/10.1016/S0015-0282(00)007160 .

5. Mcllveen M, Med B, Lok D, Pritchard J, Lashen H. Modern embryo transfer catheter and pregnancy outcome: a prospective randomized trial. Fertility and Sertility. 2005;84(4):9971000. PMID: 16213855. Available from: https://doi.org/10. 1016/j.fertnstert.2005.06.018.

6. Rhodes L, Higdon L, Boone R. Comparsion of pregnancy rates for two catheters February. Fertility and Sertility. 2007;87(2):411-416. PMID: 17094973. Available from: https: //doi.org/10.1016/j.fertnstert.2006.06.030.

7. Abou-Setta A, Al-Inany H, Mansour R, Serour G, Aboulghar M. Soft versus firm embryo transfer catheters for assisted reproduction: a systematic review and meta-analysis. Hum Reprod. 2005;20(11):3114-3121. PMID: 16040620. Available from: https://doi.org/10.1093/humrep/dei198.

8. Ata B, Isiklar A, Balaban B, Urman B. Prospective randomized comparison of Wallace and Labotect embryo transfer catheters. Reprod Biomed Online. 2007;14(4):471-476. PMID: 17425830. Available from: https://doi.org/10.1016/S14726483(10)60895-4.

9. Mcllveen M, Med B, Lok D, Pritchard J, Lashen H. Modern embryo transfer catheter and pregnancy outcome: a prospective randomized trial. Fertility and Sertility. 2005;84(4):9971000. PMID: 16213855. Available from: https://doi.org/10. 1016/j.fertnstert.2005.06.018. 
10. Zhan $Y$, Vansteelanadt $S$, Elst V, Coetsier T, Dhont M, Sutter D. The efficacy of the catheter in IVF and ICSI is operator dependent : a randomized clinical trial. Human Reproduction. 2009;24(4):880-887. PMID: 19095665. Available from: https://doi.org/10.1007/s11606-009-0976-y.

11. Rhodes L, Higdon L, Boone R. Comparsion of pregnancy rates for two catheters Fertility and Sertility. Fertility and Sertility;87(2):411-416. PMID: 17094973. Available from: https://doi.org/10.1016/j.fertnstert.2006.06.030.

12. Wood C, McMaster R, Rennie G, Trounson A. Factors influencing pregnancy rates following in vitro fertilization and embryo transfer. Fertil Steril. 1985;43:425-250.
13. Saldeen $P$, Abou-Setta M, Bergh T. A prospective randomized controlled trial comparing two embryo transfer catheters in an ART program. Fertility and Sterility. 2008;90(3):599603. PMID: 18054000. Available from: https://doi.org/10.1016/ j.fertnstert.2007.06.085.

14. Egbase P, Sharhan M, Othman S, Mutawa M. Incidence of microbial growth from the tip of the embryo transfer catheter after embryo transfer in relation to clinical pregnancy rate following in-vitro fertilization and embryo transfer. Hum Reprod. 1996;11(8):1687-1689. PMID: 8921117. Available from: https://doi.org/10.1093/oxfordjournals.humrep.a019470. 